\title{
Candida albicans Immunotherapy for Verrucae Plantaris
}

\author{
Tracey C. Vlahovic, DPM* \\ Samuel Spadone, DPM* \\ S. Patrick Dunn, DPM† \\ Tara Fussell, DPM¥ \\ Ian Hersh, DPM§ \\ Tyson Green, DPMII \\ Jeff Merrill, DPMף \\ Payel Ghosh, DPM* \\ Tran Tran, DPM*
}

\begin{abstract}
Background: Verrucae plantaris is a viral disease caused by human papilloma virus that is commonly seen in the office setting and is often challenging to treat owing to its high recurrence rate and recalcitrant profile. Candida albicans intralesional injections have been hypothesized to incite an immunogenic response toward the virus.
\end{abstract}

Methods: We report on the immunotherapeutic effect of intralesional injection of $C$ albicans into plantar verrucae with a retrospective medical record analysis of 80 patients. Using a luer-lock syringe, 0.1 to $0.3 \mathrm{~mL}$ of $C$ albicans antigen was injected into either the first known lesion or the largest lesion.

Results: The success rate of intralesional $C$ albicans, defined as total clearance of the lesion, was $65 \%$, which may be underestimated because patients lost to follow-up were included in the $35 \%$ failure rate. It was also found that female patients with a previous tissue-destructive treatment process were more than four times more likely to respond to $C$ albicans therapy, whereas this effect was less pronounced in the male patient population.

Conclusions: These results indicate that a series of intralesional injections of $C$ albicans is an effective and efficient method of treatment for verrucae plantaris. ( $\mathrm{J}$ Am Podiatr Med Assoc 105(5): 395-400, 2015)

Plantar warts (or verrucae plantaris) are likely to develop in the 2- to 12-year-old age group and occur in both sexes with equal frequency. ${ }^{1}$ Caused by direct contact with individuals with infected lesions

*Temple University School of Podiatric Medicine, Philadelphia, PA. Dr. Ghosh is now with St. Luke's Hospital, Allentown, PA. Dr. Tran is now with Metrowest Medical Center, Framingham, MA.

$\dagger$ Foot and Ankle Clinic of Oakwood Inc, Oakwood, GA. $\ddagger$ Orlando Foot and Ankle Clinic, Orlando, FL.

§Ani Orthopaedic Group of the Orthopaedic \& Spine Institute of New Jersey, Hazlet, NJ. Dr. Hersh is now with Foot and Ankle Associates of Florida, Altamonte, FL. LA.

||Imperial Health Center for Orthopaedics, Lake Charles,

ๆPodiatry Department, Skylakes Medical Center, Klamath Falls, OR. Dr. Merrill is now with Klamath Falls Foot and Ankle, LLC, Klamath Falls, OR.

Corresponding author: Tracey C. Vlahovic, DPM, Temple University School of Podiatric Medicine, $148 \mathrm{~N}$ 8th St, Philadelphia, PA 19107. (E-mail: traceyv@temple.edu) or indirectly by contact with contaminated surfaces, plantar warts are the result of human papillomavirus (HPV) infecting the basal keratinocytes of the epidermis. $^{1}$

Because no single treatment technique and no specific antiviral agents have been developed, plantar warts are a therapeutic challenge for clinicians and patients. Different modalities described in the literature range from tissue keratolysis (salicylic acid and dichloroacetic/trichloracetic acid) to immunotherapy (bleomycin) to tissue destruction (cryotherapy and surgical excision). ${ }^{2}$

Candida albicans as injection therapy for verruca vulgaris and verruca plantaris was first described in 2001. ${ }^{1}$ Treatment with $C$ albicans has been described as an extremely effective technique in that it takes two to four injections to achieve clearance. ${ }^{1-8}$ The key element of the treatment is the unique 
nature of the antigen. Candida albicans is classified as a dimorphic fungus: at normal room temperatures it takes on the form of yeast and reproduces by budding, whereas in a physiologic environment it may take on the form of hyphae or pseudohyphae. ${ }^{9}$ As a member of the normal human flora, it can be present in the mouth, throat, intestines, or genitourinary tract and rarely has any effect on nonimmunocompromised humans. ${ }^{9}$ The fungus usually can become harmful when an individual is immunosuppressed, the normal body flora is disrupted (usually by antibiotics or corticosteroids), or a change in physiology occurs. At this point, $C$ albicans will change from its yeast form into a mycelial fungal form and begin to invade the body. The microorganism has been associated with causing thrush, esophagitis, vaginal yeast infections, and deep candidiasis (Candida sepsis). ${ }^{9}$

Use of the $C$ albicans skin antigen for the treatment of verrucae is effective in a healthy individual owing to the body's recognition of the yeast as a harmful invader. ${ }^{1-8}$ Because the microorganism causes a drastic immune response in most healthy individuals, it is thought that the antigen can be used to deceive the body into subsequently killing the HPV strain as it attempts to rid the overabundance of $C$ albicans. This immunogenic response is accompanied by proliferation of peripheral blood mononuclear cells that target the HPV, suggesting that an HPV-directed cell-mediated immune response is involved in wart destruction. ${ }^{5}$

Although the amount of clinical data regarding the specific use of $C$ albicans on plantar verrucae is scarce, there have been numerous studies tracking its effectiveness on more proximal anatomical locations. One study compared the intralesional skin test antigen injection of at least one nongenital wart with that of cryotherapy. ${ }^{1}$ The patients were first tested to see whether they possessed immunity to mumps and $C$ albicans by receiving bilateral intradermal arm injections of commercial test antigen preparations. Erythema and induration of at least $5 \mathrm{~mm}$ in diameter was used as the guideline of a positive immune response. ${ }^{1}$ Those who presented with an immune response received cryotherapy or an intralesional injection of either mumps or $C$ albicans, and nonresponders received cryotherapy only. The volume of intralesional injection of mumps or $C$ albicans was determined by the size of the test reaction: $0.3 \mathrm{~mL}$ was injected with a test site induration of 5 to $20 \mathrm{~mm}, 0.2 \mathrm{~mL}$ was injected with a test site induration of 21 to $40 \mathrm{~mm}$, and $0.1 \mathrm{~mL}$ was injected with a test site induration of greater than $40 \mathrm{~mm} .{ }^{1}$ Cryotherapy and immuno- therapy were repeated every 3 weeks until complete clearing of the targeted wart was attained or for a maximum of three treatments. ${ }^{1}$ Therein, a protocol for skin testing of the antigen was established.

Determining whether immunity testing was necessary before treatment, Johnson et $\mathrm{al}^{2}$ reported that "the body is capable of ridding HPV by injection of the skin test antigen in approximately 4 treatments whether or not one skin tests."(p265) Furthermore, they found it to be more cost-effective and agreeable to the patients to skip the preliminary skin tests. ${ }^{5}$ Not only were the tests unnecessary regarding uncovering unsusceptible patients but they also proved to be useless because anergic patients are still capable of responding to intralesional injections of the antigens. ${ }^{5}$

Although the exact mechanism of action of intralesional injections of antigen has not been properly determined, there have been a few proposals. Kim et $\mathrm{al}^{10}$ believe that $C$ albicans may function by enhancing anti-HPV T-cell responses. They found that the HPV-57 L1 peptide was most commonly detected in patients whose verrucous lesions went on to clear. ${ }^{10}$ Brunk $^{11}$ felt that the immunotherapeutic agent leads to proliferation of HPV-specific mononuclear cells that mediated immunologic attack against warty tissue.

Few studies have reported on the efficacy of intralesional $C$ albicans injections to date, although preliminary data have supported the use of intralesional $C$ albicans injections.

Signore ${ }^{12}$ reviewed one of the largest studies to date with 277 patients, treating each patient with either Candida or an alternative therapy. Eightyseven of the patients were treated with Candida, and they found that 44 (51\%) exhibited complete clearance and 35 (40\%) exhibited partial clearance, leaving eight (9\%) to show no improvement. Separately, 27 patients (31\%) exhibited clearance at a distant site. ${ }^{12}$

Phillips et $\mathrm{al}^{13}$ reviewed patients clinically and also accounted for satisfaction with their outcomes based on a survey. Of 149 adult patients, they found that 107 (72\%) achieved complete clearance without any severe adverse effects with $C$ albicans treatment. Surveys showed that $76 \%$ of patients were very happy, $10 \%$ were happy, $12 \%$ were unhappy, and $2 \%$ were very unhappy with their results. ${ }^{13}$

Johnson et $\mathrm{al}^{1}$ revealed that 29 of 39 patients (74\%) had complete clearing of the targeted lesion with the immunotherapy regimen. Fourteen of the 18 patients (78\%) who demonstrated complete resolution of their immunotherapy-treated wart also showed resolution of untreated, remote warts. ${ }^{1}$ The 
study demonstrated that $C$ albicans may, in fact, serve as an effective treatment for targeted HPVinduced warts as well as distant "untreated" warts in some individuals.

Johnson et $\mathrm{al}^{2}$ published a single-blind, randomized, controlled experiment comparing immunotherapy, immunotherapy plus intralesional interferon $\alpha$ $2 \mathrm{~b}$, intralesional interferon $\alpha-2 b$ alone, and normal saline. They showed that patients who received an individual antigen of either $C$ albicans, mumps, or Trichophyton had a significantly greater positive response than the control group receiving a saline injection. ${ }^{2}$ Those who received one of the antigens (with or without interferon $\alpha-2 b$ ) showed a $56 \%$ improvement compared with injection of saline or interferon $\alpha-2 b$ alone, which produced a $23 \%$ improvement $(P<.001) .^{2}$ When the three antigens- $C$ albicans, mumps, and Trichophytonwere compared, no statistically significant differences among the antigens were reported. ${ }^{2}$ Positive results, defined as at least $5 \mathrm{~mm}$ of induration, were shown in $59 \%$ of the patients receiving only $C$ albicans, $51 \%$ receiving mumps, and $62 \%$ receiving only Trichophyton. ${ }^{2}$ Of the 57 patients who received immunotherapy and showed complete resolution of the wart treated, 21 patients, who had more than one wart at the start of the study, experienced complete resolution of all distant warts that were not intralesionally injected. ${ }^{2}$ The researchers documented that as many as 62 warts on a single patient (both treated and distant) were completely resolved after intralesional injection of antigen. ${ }^{5}$

Clifton et $\mathrm{al}^{6}$ evaluated a pediatric population of 47 patients using either Candida or mumps antigen. They found that 22 patients (47\%) achieved complete resolution; 16 patients (34\%) additionally displayed an increased $25 \%$ improvement in their symptoms. Overall, 16 patients (34\%) showed resolution at distant sites that had not been injected. ${ }^{6}$

Maronn et $\mathrm{al}^{14}$ found that there is evidence of efficacy of $C$ albicans on molluscum contagiosum and plantar verrucae-affected pediatric patients. This study supports the efficacy of $C$ albicans antigen on plantar verrucae because 48 of 55 warts (87\%) resulted in complete clearance without any serious adverse effects. Three of 55 patients additionally had partial clearance. Note that of the patients included in this paper, 21 were simultaneously treated with tissue-destructive agents, such as liquid nitrogen, salicylic acid, laser, or other possibilities. ${ }^{14}$

Majid and Imran ${ }^{15}$ recently reviewed their results after reviewing the treatment of whole-body verru- cae with intralesional injections of $C$ albicans. They included 40 patients, and 34 completed their study protocol, which included a three-injection series, intermittently giving a single injection every 3 weeks and then follow-up for 6 months to evaluate for recurrence. They found that 19 patients (56\%) achieved complete resolution, and an additional two patients (6\%) partially resolved. At the 6-month follow-up, they found that no patients had relapsed. In addition, they found that if the patient had not responded by the first or second dose of the threedose series, they were less likely to respond after the third and, therefore, they would not inject more than three times. ${ }^{15}$

Very few serious complications have been reported from any previous studies. Two separate case studies have been published introducing potential complications resulting in the use of Candida antigen. Perman et $\mathrm{al}^{16}$ published a case report of a 20-year-old woman who, after receiving a treatment, experienced a "purple digit." They found that the patient had a combination compartment syndrome in her finger and a purple discoloration due to vascular compromise initially. In a later correspondence, the authors clarified that they believed it was not tissue necrosis but a delayed hypersensitivity reaction to the antigen itself and expressed the importance of titrating the agent based on the initial response; they did not attribute the compartment syndrome to the antigen ultimately. ${ }^{16,17}$ In addition, from the pediatric population, Wilmer et $\mathrm{al}^{18}$ reported on an 8-year-old girl with a history of vitiligo and lichen sclerosus. The patient's singular verrucous lesion was treated with multiple modalities of treatment and, ultimately, an intralesional injection of $C$ albicans. This resulted in resolution of the verrucous lesion but left the region depigmented within 6 weeks, and it remained depigmented at 3-month follow-up. ${ }^{18}$ Although no fatal adverse effects have been reported to date, it is important to consider the novelty of the treatment and carefully observe for any significant changes, such as a hypersensitivity response or changes in pigmentation. Overall, the risks of this treatment are relatively low, and other factors should be reviewed before implementing this modality.

A final concern is that of the financial advantages and disadvantages of a multimodal treatment, including intralesional $C$ albicans injections. Clemons et $\mathrm{al}^{8}$ performed a comparative cost analysis comparing the seven most common interoffice treatment modalities for the care of plantar verrucae. They found that in terms of cost efficacy, $C$ albicans was second only to a carbon dioxide laser 
taking into account the number of visits required for eradication, physician costs required to implement the treatment, and medication costs. Owing to the variety of adverse effects associated with the carbon dioxide laser, $C$ albicans may, overall, be most cost efficient. In addition, the study found that owing to the frequent office visits associated with cryotherapy, or liquid nitrogen tissue destruction, the total cost in dollars triples that of intralesional $C$ albicans injections. Ultimately, this study concluded that intralesional $C$ albicans injections are underused in the care of plantar verrucae in the office setting, although it is important to note that the investigators did not take into account recurrence of plantar verrucae.

Overall, promising results have been reported throughout the dermatologic literature regarding the efficacy, safety, and cost-effectiveness of $C$ albicans on the treatment of verrucae plantaris, although no formal study has ever been published in the podiatric medical literature. After a series of successful treatments using intralesional $C$ albicans, the decision was made to formally study the results in the form of a retrospective medical record review.

\section{Methods}

An institutional review board-approved retrospective medical record review was undertaken, examining all patients treated by the senior author (T.C.V.) for pedal verrucae between January 1, 2004, and December 31, 2007. Patients who did not receive $C$ albicans skin test antigen injection were excluded from the review. The individual progress notes were examined for objective clinical findings, diagnosis, and treatment. Objective signs for verruca were papular or planar hyperkeratotic lesions that exhibited thrombosed capillaries, pinpoint bleeding on debridement, and an interruption of skin lines traversing the lesion after debridement.

Pertinent patient information, including patient name, medical record number, age, sex, location of verruca, previous treatments, number of Candida injections, total number of treatments, amount of Candida injected, and time to clearance/failure were recorded. Time to clearance was defined as objective physical examination findings (skin lines present throughout lesion, lack of thrombosed capillaries, and resolution of hyperkeratotic lesions). Failure of treatment was defined as continuance of pain in the location of verruca, the presence of thrombosed capillaries in the lesion, and lack of skin lines traversing the lesion after debridement.

The technique of the treatment was intradermal injection of the $C$ albicans skin test antigen (Nielsen BioSciences, San Diego, California) at the site of either the first known lesion ("mother wart") or the largest lesion on the foot. This was done using a 1$\mathrm{cm}^{3}$ tuberculin syringe with a 30-gauge half-inch needle at a $15^{\circ}$ angle to the skin. The amount of 0.1 to $0.3 \mathrm{~cm}^{3}$ injected into a singular lesion was determined by the size and extent of the lesions. As significant back pressure was typically encountered during injection, a luer-lock syringe and a slow injection technique $(>30 \mathrm{sec})$ were used. Patients who received injection therapy were scheduled to return at 1-month intervals.

Follow-up was continued until the previously mentioned criteria for clearance were met. A minimum of two attempts to contact patients by telephone who did not attend follow-up visits was performed by the contributing authors. Questions that were asked included the following: if the lesion had resolved, length of time between last treatment and resolution; was the patient satisfied with the treatment rendered; and would the patient undergo treatment again if another verruca developed.

\section{Results}

This retrospective medical record review of patients treated with $C$ albicans at the Temple University Foot and Ankle Institute (Philadelphia, Pennsylvania) between 2004 and 2007 for plantar verruca was conducted to estimate the clearance rate for this therapy. The review identified 80 patients, 51 women and 29 men. The mean patient age was 32.8 years, with no significant differences associated with sex (mean: 32.27 years for women vs 33.66 years for men).

It was not possible to determine how long the verrucae were present in many cases because in some cases the patients sought care at other providers before coming to the Foot and Ankle Institute. The mean number of treatments received at the Foot and Ankle Institute varied widely, with a mean $\pm \mathrm{SD}$ of $4.68 \pm 3.54$. No significant difference in the mean number of treatments received was noted when comparing the sexes.

Lesions were classified as having cleared when skin lines became present throughout the lesion and there was a lack of thrombosed capillaries or resolution of hyperkeratotic lesions. Patients whose lesions did not meet these criteria and those lost to follow-up were classified as having failed therapy. 
Although classifying all patients lost to follow-up as failing therapy could deflate estimates of therapeutic efficacy, this approach was selected as the most conservative option. On this basis, $65 \%$ of the patients were classified as having been treated successfully (95\% confidence interval [CI], 0.540.745), with the remaining $35 \%$ (95\% CI, 0.255-0.459) being classified as treatment failures.

When clearance rates in men and women were estimated separately, men exhibited a slightly higher clearance rate, but this difference was not significantly different (likelihood ratio $\chi^{2}=2.432 ; P$ $=.1188$ ). At this point, an incidental note was made that patients who had received treatment that included a tissue-destructive modality before $C$ albicans injection seemed more likely to clear than those who had not, and it was decided to formally test the hypothesis that clearance rates in those with previous exposure to tissue-destructive therapy were higher than those in patients without previous exposure.

The two-tailed likelihood ratio comparing clearance in patients with histories of exposure to tissuedestructive therapies with that in patients without such previous treatment yielded a Pearson $\chi^{2}$ of 11.924 , with an associated $P=.0006$. The (left) onesided Fisher exact test in favor of higher clearance rates in those previously treated with tissuedestructive therapies was also computed, and a $P$ $=.001$ was obtained. The relative risk of clearance in patients with previous tissue-destructive therapy compared with that in patients without previous tissue-destructive therapy was estimated to be 2.769 (95\% CI, 1.182-6.489). These results are depicted graphically in the mosaic plot in Figure 1.

The Cochran-Mantel-Haenszel statistic for general association of categories was calculated to

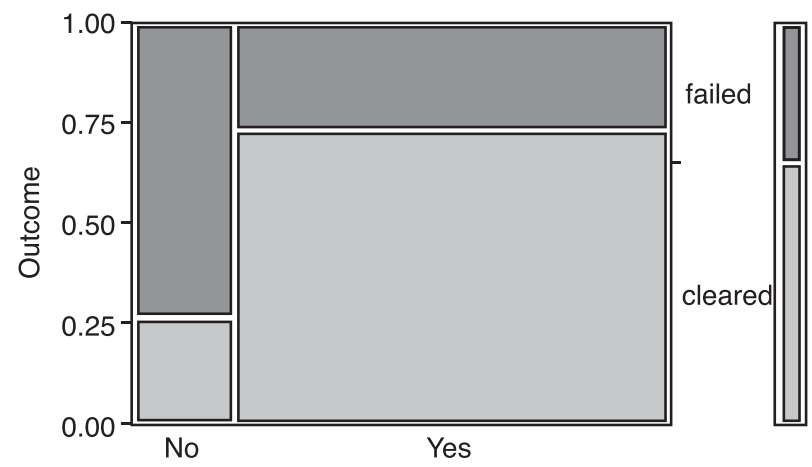

Figure 1. The relative risk of clearance in patients with previous tissue-destructive therapy compared with that in patients without previous tissue-destructive therapy. stratify the observed difference by sex, yielding a $\chi^{2}$ of 10.247 , with a corresponding $P=.0014$. When evaluation of the frequency of clearance by history expected under the hypothesis of independence versus that observed in this study was performed separately for each sex, the likelihood ratio $\chi^{2}$ was determined to be 11.890 for women and 0.145 for men, with corresponding $P=.006$ and $P=.704$, respectively. When the relative risks of clearance in those with and without a history of previous exposure to tissue-destructive treatment were evaluated separately for each sex, the relative risk was estimated to be 4.308 (95\% CI, 1.197-15.499) for women versus 1.154 (95\% CI, 0.504-2.639) for men. This suggests that the improvement in overall clearance rates with previous tissue-destructive therapy was largely due to the beneficial effect of the latter in female patients.

\section{Discussion}

Treatment of plantar verrucae using an intralesional injection of skin antigen, namely, $C$ albicans, presents an efficient and cost-effective alternative treatment to the current standard of care, and this is a concept that has been demonstrated in recent literature.

Because this study is retrospective, there are certain limitations. For example, there is no true control group that allows added comparison with a group receiving neither $C$ albicans nor tissuedestructive treatment. This is an issue that can be remedied in the future with the proper application of a planned prospective study.

This study was a retrospective analysis of 80 patients with plantar verrucae seen between 2004 and 2007 at the Foot and Ankle Institute of Philadelphia. The intention of the study was to analyze whether intralesional $C$ albicans injections are an effective modality of treatment for plantar verrucae. The mean age of the present patient population was approximately 33 years and had no effect on the efficacy of treatment statistically. It was found that the mean number of treatments required was 4.68 and resulted in a $65 \%$ success rate, whereas the $35 \%$ failure rate included those who failed treatment based on preset parameters and those lost to follow-up, indicating that the success rate may be underestimated. In addition, it was found that those who had received a previous treatment of a tissue-destructive process were more likely to clear by 2.769 times. When this patient population was further subdivided by sex it was found that women, especially, were more likely to 
clear when an intralesional $C$ albicans injection followed a treatment using a tissue-destructive process by 4.308 times. This leads to the conclusion that there is a likely benefit to multimodal therapy involving initial use of a tissue-destructive process, such as Cantharone (Dormer Laboratories Inc, Toronto, Ontario, Canada), followed by an intralesional injection of $C$ albicans. This study provides evidence that this effective therapy is a reasonable modality of treatment for plantar verrucae. To more accurately evaluate the efficacy of $C$ albicans, there is need for further prospective studies in the future.

Financial Disclosure: None reported.

Conflict of Interest: None reported.

\section{References}

1. JoHnson SM, RoBerson PK, Horn TD: Intralesional injection of mumps or Candida skin test antigens: a novel immunotherapy for warts. Arch Dermatol 137: 451, 2001.

2. Johnson S, Roberson P, Horn T: Intralesional immunotherapy of warts with mumps, candida, and Trichophy ton skin test antigens. Arch Dermatol 141: 589, 2005.

3. Kwok CS, Gibbs S, Bennett C, et AL: Topical treatments for cutaneous warts. Cochrane Database Syst Rev 9: CD001781, 2012.

4. Bunney MH, Nolan MW, Williams DA: An assessment of methods of treating viral warts by comparative treatment trials based on a standard design. Br J Dermatol 94: 667,1976 .

5. JoHnson S, HoRn T: Intralesional immunotherapy for warts using a combination of skin test antigens: a safe and effective therapy. J Drugs Dermatol 3: 263, 2004.

6. Clifton MM, Johnson SM, Roberson PK, ET AL: Immuno- therapy for recalcitrant warts in children using intralesional mumps or Candida antigens. Pediatr Dermatol 20: $268,2003$.

7. BACELIERI R, Johnson SM: Cutaneous warts: an evidencebased approach to therapy. Am Fam Physician 72: 647, 2005.

8. Clemons RJ, Clemons-Miller A, Johnson SM, et AL: Comparing therapy costs for physician treatment of warts. J Drugs Dermatol 2: 649, 2003.

9. LEUNG L: Hyfrecation for recalcitrant nongenital warts. J Family Med Prim Care 2: 141, 2013.

10. Kim KH, Horn TD, Pharis J, ET AL: Phase 1 clinical trial of intralesional injection of Candida antigen for the treatment of warts. Arch Dermatol 146; 12. 2010.

11. BRUNK D: Injection of Candida antigen works on warts. Skin Allergy News 30: 5, 1999.

12. Signore RJ: Candida albicans intralesional injection immunotherapy of warts. Cutis 70: 185, 2002.

13. Phillips RC, Ruhl TS, Pfenninger JL, et Al: Treatment of warts with Candida antigen injection. Arch Dermatol 136: $1274,2000$.

14. Maronn M, Salm C, Lyon V, ET aL: One year experience with Candida antigen immunotherapy for warts and molluscum. Pediatr Dermatol 25: 189, 2008.

15. MAJID I, ImRAN S: Immunotherapy with intralesional Candida albicans antigen in resistant or recurrant warts: a study. Indian J Dermatol 58: 360, 2013.

16. Perman M, Sterling JB, Gaspari A: The painful purple digit: an alarming complication of Candida albicans antigen treatment of recalcitrant warts. Dermatitis 16: 38, 2005.

17. SignORE RJ: An alarming complication of Candida albicans antigen treatment of recalcitrant warts [letter to the editor]. Dermatitis 16: 216, 2005.

18. Wilmer EN, Burkhart CN, Morrell DS: Goodbye warts, hello vitiligo: Candida antigen-induced depigmentation. Pediatr Dermatol 30: e214, 2013. 